\title{
Screening for peripheral artery disease in dialysis patients: an opportunity for early disease detection and timely initiation of appropriate therapeutic measures
}

\author{
Kosmas I. Paraskevas • Sotirios A. Koupidis • \\ Alexandros A. Tzovaras • Achilleas Nikolaou • \\ Dimitri P. Mikhailidis
}

Received: 13 November 2010/Accepted: 21 December 2010/Published online: 6 January 2011

(C) Springer Science+Business Media, B.V. 2011

Otsubo et al. [1] in this issue of International Urology and Nephrology report that peripheral arterial disease (PAD) is an independent predictor of mortality in hemodialysis patients. In univariate analysis, the presence of PAD was associated with a $67 \%$ increase in long-term mortality risk in patients undergoing maintenance hemodialysis compared with the absence of PAD (odds ratio [OR], 1.67; 95\% confidence interval [CI], 1.18-2.28; $P=0.004$ ) [1]. The relation between PAD and mortality persisted even after adjustment for age, gender, duration of dialysis, diabetes mellitus and smoking (OR, 1.575;

K. I. Paraskevas $(\bowtie) \cdot$ A. Nikolaou

Department of Vascular Surgery, "Red Cross" Hospital, 24, Al. Papagou street, Athens, Greece

e-mail: paraskevask@hotmail.com

\section{S. A. Koupidis}

Department of Dermatology, University of Athens

Medical School, Athens, Greece

A. A. Tzovaras

1st Department of Oncology, Aghios Savvas Hospital, Athens, Greece

D. P. Mikhailidis

Department of Clinical Biochemistry (Vascular Disease

Prevention Clinics), Royal Free Hospital Campus,

University College London Medical School,

University College London (UCL), London, UK
95\% CI, 1.038-2.381; $P=0.033$ ) [1]. These findings support the results of earlier studies showing that PAD is common in dialysis patients and that it is associated with increased cardiovascular morbidity and mortality rates in this population [2-4]. This Editorial discusses some additional aspects that may be of interest.

There is a strong two-way correlation between impaired renal function and PAD. A recent comprehensive review demonstrated that $27-36 \%$ of patients with PAD also have concomitant impaired renal function [5]. The presence of impaired renal function in these individuals is associated not only with higher morbidity and mortality rates but also with the occurrence of cardiovascular events (stroke, myocardial infarction, and cardiovascular death) [5]. Furthermore, impaired renal function negatively affects survival, limb salvage and graft patency rates when these individuals undergo lower extremity revascularization procedures [5]. Conversely, patients on chronic dialysis have high atherosclerosis and arteriosclerosis rates [6]; the increased arterial stiffness is responsible for the high prevalence of carotid artery stenosis, myocardial infarction, PAD, and stroke in this population [6]. PAD is an atherosclerotic occlusive disease with/without vascular calcifications in the intima of lower extremity arteries [7-9]. The symptoms of PAD include intermittent claudication, which progresses to ischemic leg pain, critical limb ischemia, and finally, tissue necrosis requiring amputation. The prevalence of PAD is high among patients 
with chronic kidney disease or end-stage renal disease [7-9]. Patients with end-stage renal disease have nearly 10 -fold higher lower extremity amputation rates compared with the general population $[8,9]$. Cardiovascular disease is the leading cause of death in both chronic kidney disease and peritoneal dialysis/hemodialysis patients [10]. It was recently emphasized that several vascular disease prevention measures (including smoking cessation, and control of hyperphosphatemia, body weight, and blood pressure) should be initiated to reduce cardiovascular events and mortality in this high-risk population [10]. This is the reason why dialysis patients are commonly prescribed cardiovascular medications (e.g. statins, clopidogrel, acetylsalicylic acid, beta-blockers, and angiotensin-converting enzyme [ACE] inhibitors) despite the poor level of direct evidence of benefit for some of these drugs [11]. Indeed, multicentre randomized trials have failed to show a benefit of statin therapy in dialysis patients [12, 13], thus suggesting that cardiovascular disease in patients undergoing hemodialysis is different than in other patients $[14,15]$.

Otsubo et al. [1] used ankle-brachial index (ABI) to screen their patients for the presence of subclinical PAD. ABI is not only a marker of both PAD and systemic atherosclerosis but also a predictor of vascular events [16]. A recent study, however, disputed the value of $\mathrm{ABI}$ for the detection of PAD in dialysis patients [17]. This cross-sectional outpatient cohort study examined the prevalence of PAD among hemodialysis patients using both the ABI and duplex ultrasonography of the lower limbs arteries.

Although the specificity of ABI values for PAD was $94.8 \%$, the sensitivity of this test was as low as $49.0 \%$ in this population. It was thus concluded that although $\mathrm{ABI}$ is a simple and reliable test for PAD screening in the general population, its sensitivity decreases considerably in dialysis patients [17]. Another recent study assessed the value of vascular calcification scores on plain radiographs of the lower limb arteries for the prediction of PAD in 102 patients with chronic kidney disease (24 pre-dialysis, 58 hemodialysis, and 20 peritoneal dialysis individuals) [7]. Patients with high vascular calcification scores demonstrated more atherosclerotic calcified plaques in the femoral and popliteal arteries. Furthermore, the prevalence of PAD in these patients was strongly associated with high vascular calcification scores $(P<0.01)$. The conclusion reached was that a high vascular calcification score was a strong predictor for the presence of PAD (OR, 6.66; $P<0.001)$ [7]. Thus, in dialysis patients, high vascular calcification scores on plain radiographs [7] or duplex ultrasonography of the lower limb arteries [17] may be more accurate predictors of PAD than the ABI. Due to the strong association between PAD and increased cardiovascular mortality rates in dialysis patients [1-4], the early detection of subclinical PAD in these patients is crucial. The early recognition of subclinical PAD will allow the timely initiation of preventive measures (e.g. statins and antiplatelet agents). Statins not only improve renal function $[18,19]$ but also reduce the high cardiovascular mortality and morbidity rates seen in those individuals [20].

In the last few years, a few alternative options have emerged other than the traditional in-center hemodialysis or peritoneal dialysis, such as home dialysis [21]. These alternative dialysis options may have the disadvantage of relatively infrequent medical supervision and patient follow-up. Consequently, subclinical PAD in these patients may escape diagnosis. As a result, initiation of appropriate vascular disease prevention measures (i.e. body weight reduction, smoking cessation, antiplatelet medication, and lipid-lowering treatment with statins) may not be timely implemented. The delay in the establishment of the proper diagnosis and the subsequent delay in the implementation of routine statin and antiplatelet treatment may in turn result in increased mortality and morbidity rates.

In conclusion, screening and early detection of PAD in dialysis patients is crucial and should be coupled with timely initiation of preventive measures to reduce the high cardiovascular morbidity and mortality rates seen in this population. There is recent evidence suggesting that the ABI may not be a sensitive enough test for the diagnosis of PAD in dialysis patients [17]. In these patients, duplex ultrasonography [17] or vascular calcification scores [7] of the lower extremity arteries may be required. Nephrologists should have a high index of suspicion for the early detection of PAD in dialysis patients to ensure the timely initiation of the appropriate preventive measurements for the reduction in the high cardiovascular mortality and morbidity rates seen in this population. 


\section{References}

1. Otsubo S, Kitamura M, Wakaume T et al. (2011) Association of peripheral artery disease and long-term mortality in hemodialysis patients. Int Urol Nephrol (in press)

2. O'Hare A, Johansen K (2001) Lower-extremity peripheral arterial disease among patients with end-stage renal disease. J Am Soc Nephrol 12:2838-2847

3. Okamoto K, Oka M, Maesato K et al (2006) Peripheral arterial occlusive disease is more prevalent in patients with hemodialysis: comparison with the findings of multidetector-row computed tomography. Am J Kidney Dis 48:269-276

4. Rajagopalan S, Dellegrottaglie S, Furniss AL et al (2006) Peripheral arterial disease in patients with end-stage renal disease: observations from the Dialysis Outcomes and Practice Patterns Study (DOPPS). Circulation 114:1914-1922

5. Paraskevas KI, Giannoukas AD, Mikhailidis DP (2009) Renal function impairment in peripheral arterial disease: an important parameter that should not be neglected. Ann Vasc Surg 23:690-699

6. Kanbay M, Afsar B, Gusbeth-Tatomir P, Covic A (2010) Arterial stiffness in dialysis patients: where are we now? Int Urol Nephrol 42:741-752

7. An WS, Son YK, Kim SE et al (2010) Vascular calcification score on plain radiographs of the feet as a predictor of peripheral arterial disease in patients with chronic kidney disease. Int Urol Nephrol 42:773-780

8. Eggers PW, Gohdes D, Pugh J (1999) Nontraumatic lower extremity amputations in the medicare end-stage renal disease population. Kidney Int 56:1524-1533

9. O'Hare A, Johansen K (2001) Lower-extremity peripheral arterial disease among patients with end-stage renal disease. J Am Soc Nephrol 12:2838-2843

10. Paraskevas KI, Kotsikoris I, Koupidis SA et al (2010) Cardiovascular events in chronic dialysis patients: emphasizing the importance of vascular disease prevention. Int Urol Nephrol 42:999-1006
11. Sood MM, Battistella M, Lok CE (2009) Patterns of cardioprotective medication prescription in incident hemodialysis patients. Int Urol Nephrol 41:1021-1027

12. Wanner C, Krane V, März W, German Diabetes, Dialysis Study et al (2005) Atorvastatin in patients with type 2 diabetes mellitus undergoing hemodialysis. N Engl J Med 353:238-248

13. Fellström BC, Jardine AG, Schmieder RE, AURORA Study Group et al (2009) Rosuvastatin and cardiovascular events in patients undergoing hemodialysis. N Engl J Med 360:1395-1407

14. Herzog CA, Mangrum JM, Passman R (2008) Sudden cardiac death and dialysis patients. Semin Dial 21:300-307

15. Guérin AP, Pannier B, Marchais SJ et al (2008) Arterial structure and function in end-stage renal disease. Curr Hypertens Rep 10:107-111

16. Paraskevas KI, Kotsikoris I, Koupidis SA et al (2010) Ankle-brachial index: a marker of both peripheral arterial disease and systemic atherosclerosis as well as a predictor or vascular events. Angiology 61:521-523

17. Ogata H, Kumata-Maeta C, Shishido K et al. (2011) Detection of peripheral artery disease by duplex ultrasonography among hemodialysis patients. Clin J Am Soc Nephrol (in press)

18. Alnaeb ME, Youssef F, Mikhailidis DP et al (2006) Shortterm lipid-lowering treatment with atorvastatin improves renal function but not renal blood flow indices in patients with peripheral arterial disease. Angiology 57:65-71

19. Alnaeb ME, Alobaid N, Seifalian AM et al (2006) Statins and peripheral arterial disease: potential mechanisms and clinical benefits. Ann Vasc Surg 20:696-705

20. Daskalopoulou SS, Daskalopoulos ME, Liapis CD et al (2005) Peripheral arterial disease: a missed opportunity to administer statins so as to reduce cardiac morbidity and mortality. Curr Med Chem 12:443-452

21. Oreopoulos DG, Thodis E, Passadakis P et al (2009) Home dialysis as a first option: a new paradigm. Int Urol Nephrol 41:595-605 